Relations industrielles

Industrial Relations

\title{
Responsabilité sociale des entreprises: mirage ou réalité ?, Par Mustapha Bettache (2015) Québec: Presses de l’Université de Laval, 84 pages. ISBN: 978-2-7637-2781-3
}

\section{Claire Dupont}

Volume 71, numéro 3, été 2016

URI : https://id.erudit.org/iderudit/1037671ar

DOI : https://doi.org/10.7202/1037671ar

Aller au sommaire du numéro

\section{Éditeur(s)}

Département des relations industrielles de l’Université Laval

\section{ISSN}

0034-379X (imprimé)

1703-8138 (numérique)

Découvrir la revue

\section{Citer ce compte rendu}

Dupont, C. (2016). Compte rendu de [Responsabilité sociale des entreprises: mirage ou réalité ?, Par Mustapha Bettache (2015) Québec: Presses de

l'Université de Laval, 84 pages. ISBN: 978-2-7637-2781-3]. Relations industrielles

/ Industrial Relations, 71(3), 580-582. https://doi.org/10.7202/1037671ar

Tous droits réservés @ Département des relations industrielles de l’Université Laval, 2016
Ce document est protégé par la loi sur le droit d'auteur. L’utilisation des services d'Érudit (y compris la reproduction) est assujettie à sa politique d'utilisation que vous pouvez consulter en ligne.

https://apropos.erudit.org/fr/usagers/politique-dutilisation/ 
page 169: "The analysis of time appropriation as an act of resistance turned out to be much more complicated than I had assumed ». De manière plus fondamentale, le livre montre que travailler fort n'est pas nécessairement le plus payant ( " at work, it is not what you do, but how you look like you are doing it », nous dit l'auteur), que le " travail vide » n'est pas nécessairement une mauvaise chose, mais qu'au contraire, il peut être vu comme un « travail pour soi » qui est également profitable à l'entreprise puisqu'il peut favoriser l'innovation, l'originalité et la créativité. De ce point de vue, Empty Labor est incontestablement un livre novateur qui permet de penser le travail à partir du temps de non-travail et de voir d'une façon plus positive et réaliste la dynamique complexe de l'articulation entre la subjectivité des individus et les logiques organisationnelles.

\section{Mircea Vultur}

Professeur

Institut national de la recherche scientifique Centre Urbanisation Culture Société Québec

\section{Responsabilité sociale des entreprises: mirage ou réalité ?}

Par Mustapha Bettache (2015) Québec: Presses de l'Université de Laval, 84 pages. ISBN : 978-2-7637-2781-3.

Dans cet ouvrage, Mustapha Bettache s'interroge sur les implications de la responsabilité sociale des entreprises (RSE) au sein de nos organisations, et plus globalement, de notre société. Sensée être un vecteur de bien-être collectif, la responsabilité sociale ne serait-elle pas aussi une nouvelle modalité de gouvernance du capitalisme ? C'est notamment l'une des questions posée par I'auteur qui, au fil de son ouvrage, interpelle le lecteur en l'éclairant sur certaines facettes paradoxales de la RSE: existence de normes socialement responsables, mais faiblesse de leur poids; nécessité pour les entreprises de considérer leurs parties pre- nantes, tout en devant se positionner face aux éventuelles divergences de leurs intérêts; mésentente entre le monde des affaires et les organisations internationales sur le concept de développement durable; rôle ambigu de l'État face aux entreprises; .... . Chacun des quatre chapitres de l'ouvrage tend à montrer toutes les ambiguïtés entourant la RSE et invite à s'interroger sur l'aspect réel ou illusoire de celle-ci.

Ainsi, le premier chapitre s'intéresse aux "Théories de la responsabilité sociale de I'entreprise». Revenant sur l'origine, puis sur le développement progressif de la RSE dans le contexte actuel de mondialisation, I'auteur expose ensuite différentes approches de la RSE, dont la théorie des parties prenantes. Si l'auteur rappelle que cette théorie suppose, pour l'organisation, de considérer les différents acteurs susceptibles d'être affectés par ses décisions et activités, il souligne bien le difficile arbitrage à réaliser entre les attentes éventuellement divergentes de ceux-ci. Les apports de l'École de Montréal et de l'École allemande sont ensuite évoqués. Mustapha Bettache souligne, enfin, la diversité des instruments sensés soutenir les démarches socialement responsables des entreprises. Si les normes, les "labels", les programmes spécifiques tels que Global Compact, les certifications, ..., sont nombreux, ils n'en souffrent pas moins de certaines imperfections : absence de contrôle ou d'obligations, dont le nonrespect conduirait à des sanctions; utilisation à des fins marketing; etc. .

Le deuxième chapitre s'intéresse aux relations entre la « Responsabilité sociale de I'entreprise et le développement durable». L'auteur montre bien le décalage entre la vision du développement durable dans les milieux d'affaires, articulée autour des $3 \mathrm{P}$ (People, Planet, Profit), et celle des " organisations internationales de la sphère onusienne » (Bettache, 2015 : 26), qui tendent plutôt à remplacer la notion de profit par celle de prospérité. La norme ISO 26000, qui invite à réfléchir aux impacts des activi- 
tés de l'entreprise sur l'environnement et la société, permet d'entrevoir des liens entre la RSE et le développement durable. Légitimée par grand nombre d'académiques et d'experts, elle donne ainsi des lignes directrices aux entreprises pour les aider à développer une compréhension commune de la RSE et à contribuer au développement durable. Toutefois, comme cette norme n'établit aucune procédure de contrôle ni de sanction, on peut s'interroger sur le caractère socialement responsable des entreprises qui affirmeraient la suivre. Selon Mustapha Bettache (2015:29), le développement durable «traduirait dans l'esprit de ses promoteurs, un changement de paradigme qui met l'accent sur l'incorporation du facteur écologique dans le modèle de développement économique». Mais I'auteur s'interroge: "l'adhésion à une telle vision de développement ne doit-elle pas s'accompagner de l'abandon d'une vision d'industrialisation forcenée qui a prévalu jusqu'ici et qui n'a accordé que très peu de place aux aspects écologiques? » (Bettache, 2015: 29-30). À travers des exemples relatifs à la mise au point de moteurs moins pollueurs chez les constructeurs automobiles ou à l'exploitation des sables bitumeux, I'auteur pointe du doigt la délicate position dans laquelle se retrouve l'État: bénéficier des éventuelles retombées de ces projets ou réagir contre leurs effets néfastes sur l'environnement et les populations? II évoque ensuite des expériences d'économie sociale qui pourrait constituer une alternative au capitalisme et une réaction complémentaire à celle des États, relativement affaiblis dans le contexte actuel de mondialisation.

Dans le troisième chapitre, "Responsabilité sociale, sociétale ou globale ? Principaux enjeux », I'auteur invite à concevoir les questions de responsabilité sociale dans une optique plus globale, en prenant en compte la diversité des enjeux sous-jacents. C'est que ceux-ci sont nombreux : délocalisations et pertes d'emplois qui en découlent; pratiques discriminatoires, notamment à l'égard des femmes et des travailleurs immigrants ; travail des enfants; employabilité découlant notamment des innovations technologiques de plus en plus nombreuses;... Il présente ensuite quelques initiatives RSE développées par certaines entreprises.

Enfin, le quatrième chapitre, "État fort et résistance citoyenne. Les effets pervers de la mondialisation », invite à réfléchir au rôle de l'État dans le développement du bien-être collectif et dans la protection des citoyens, face à la toute-puissance du secteur privé. Selon Mustapha Bettache, il est primordial que la RSE ne soit pas le fait d'une élite: le rôle des citoyens et des organisations nongouvernementales dans la promotion d'une société socialement responsable est lui aussi essentiel et vient compléter le rôle de l'État. Le mouvement "Stop Desabusios» apparu en 2009 au cœur de la crise espagnole montre, par exemple, que la mobilisation de milliers de citoyens indignés a permis certaines avancées, au départ inespérées, face à la problématique des évictions.

À travers ces quatre chapitres, l'ouvrage de Mustapha Bettache conduit le lecteur à réfléchir, sur base de situations paradoxales, à la RSE telle que pratiquée aujourd'hui et au rôle que chacun d'entre nous pourrait jouer dans la construction d'une société socialement responsable. Le lecteur peut parfois regretter le manque d'approfondissement ou d'argumentation de certaines pistes avancées (conception politique de la RSE à partir de la théorie de la démocratie délibérative d'Habermas, expérience chinoise, ...) ou le fait que certaines propositions, présentées comme un moyen de rendre la société plus responsable, soient finalement identifiées principalement en termes de dérives (expérience québécoise autour du commerce équitable). L'ouvrage est néanmoins interpellant et en appelle à la prudence quant au caractère socialement responsable des entreprises. Non sans pointer du doigt les comportements parfois extrêmes des entreprises face au capitalisme, il rappelle que la RSE est l'affaire de 
tous, non seulement des entreprises, mais aussi des citoyens, de l'État, des institutions locales, nationales et internationales.

\section{Claire Dupont}

Institut de recherche humanOrg

Université de Mons, Belgique

\section{Refonder le système de protection sociale. Pour une nouvelle génération de droits sociaux}

Par Bernard Gazier, Bruno Palier et Hélène Périvier (2014) Paris : Presses de Sciences Po, Coll. Nouveaux Débats, 200 pages.

ISBN : 978-2-7246-1625-5.

Cet ouvrage est le fruit d'un long processus de réflexion et d'échange sur le système de protection sociale en Europe et, plus particulièrement, en France. Dans une perspective multidisciplinaire, les auteurs proposent une nouvelle orientation politique qui vise trois objectifs : 1- améliorer le progrès social; 2- atteindre l'égalité entre les hommes et les femmes; et 3- accroître la prospérité économique. Centré sur les politiques sociales et sur l'emploi, leur livre s'inscrit dans une vision novatrice du système de protection social français via la mise en place d'une nouvelle génération de droits sociaux. II contient trois chapitres.

Dans le premier chapitre, les auteurs dévoilent les limites du système de protection sociale français. Entre 1945 et 1970, le système de protection sociale était caractérisé par deux dimensions, à savoir " familiste » et " assurantielle ». Ce système a alors contribué au progrès social en assurant un revenu aux personnes âgées et en encourageant l'augmentation de la fécondité. II a aussi permis de maîtriser les inégalités, de réduire la pauvreté et de mettre en place un système de soins de qualité. Toutefois, à partir des années 1970, l'économie de la France a connu de profonds bouleversements. Dès lors, la probabilité d'avoir un emploi s'est amoindrie. Ces facteurs ont sapé le financement du régime des protections. Face à cette conjoncture, la France a adopté une politique orientée vers la flexibilisation du marché du travail — qui est aussi considérée comme un facteur d'aggravation des inégalités salariales entre les hommes et les femmes ainsi que vers la mise au travail. Certes, des mesures et des prestations, comme l'allocation « parent isolé » (1976) et le « revenu minimum d'insertion » (1988), qui ont été remplacé en 2009 par le « revenu de solidarité active » (RSA), ont été mises en œuvre, mais leur efficacité a, cependant, diminué avec le temps; ces mesures sont donc aujourd'hui insuffisantes. En plus, le système souffre d'un manque d'accompagnement, à la fois des transitions professionnelles et de l'émancipation des individus.

Le deuxième chapitre présente une nouvelle architecture du système de protection sociale. Les auteurs proposent une nouvelle égalité fondée sur quatre principes. En s'inspirant de John Dewey et Amartya Sen, les auteurs proposent, en premier, « le principe de justice », qui renvoie à la capacité de l'individu de décider et de vivre selon ses choix. Le deuxième principe fait référence à la solidarité citoyenne et à la société d'ensemble. Ce principe devrait permettre à toutes et à tous un accès égal aux services publics et sociaux. Le troisième principe renvoie à l'investissement social en amont. Ce principe peut être considéré comme une phase préparatoire qui permettra d'équiper les personnes, de réduire les risques sociaux et d'accroître la possibilité de s'insérer sur le marché du travail. Le quatrième et dernier principe, quant à lui, fait référence à l'égalité entre les sexes. Ce principe devrait permettre une répartition égalitaire via une réorganisation de la vie sociale et économique indépendamment du sexe. En outre, les auteurs insistent sur la mise en place d'une logique d'égalité et de qualité, principalement en améliorant les politiques éducatives, les soins, la construction de logements et les transports collectifs, tout en favorisant la création d'emplois qualifiés. Aussi, les auteurs proposent de faciliter les transitions professionnelles, à savoir la formation tout au long de la vie, et I'assurance employabilité, couplées aux 